\title{
Modelling couplings among the oscillators of the cardiovascular system
}

\author{
Aneta Stefanovska $\ddagger$, Dmitrii Luchinsky† and Peter V E \\ McClintock $\uparrow$ \\ $\ddagger$ Group of Nonlinear Dynamics and Synergetics, Faculty of Electrical Engineering, \\ University of Ljubljana, Tržaška 25, 1000 Ljubljana, Slovenia. (aneta@osc.fe.uni-lj.si) \\ $\dagger$ Department of Physics, University of Lancaster, Lancaster LA1 4YB, UK. \\ (d.luchinsky@lancaster.ac.uk, p.v.e.mcclintock@lancaster.ac.uk)
}

\begin{abstract}
A mathematical model of the cardiovascular system is simulated numerically. The basic unit in the model is an oscillator that possesses a structural stability and robustness motivated by physiological understanding and by the analysis of measured time series. Oscillators with linear couplings are found to reproduce the main characteristic features of the experimentally obtained spectra. To explain the variability of cardiac and respiratory frequencies, however, it is essential to take into account the rest of the system, i.e. to consider the effect of noise. It is found that the addition of noise also results in epochs of synchronization, as observed experimentally. Preliminary analysis suggests that there is a mixture of linear and parametric couplings, but that the linear coupling seems to dominate.
\end{abstract}

PACS numbers: 87.10.+e, 5.45.Xt, 5.40.Ca, 87.19.Hh

|| To whom correspondence should be addressed. 


\section{Introduction}

Coupled oscillators are ubiquitous in nature. They have been studied intensively over the years in many areas of science and technology including e.g. physics (Haken 1983, Strogatz 1994), chemistry (Kuramoto 1984) and biology (Winfree 1980). However, most theoretical work on coupled oscillators refers either to a small number of oscillators (e.g. 2 , or at most 3 ), or to large ensembles that can be treated statistically; only in a few cases is the effect of random fluctuations included explicitly.

It has long been known that the heart of a healthy human subject in repose does not beat regularly. The rhythmic variation in the heart rate occurring at the frequency of respiration, known as respiratory sinus arrhythmia, has been the most studied cardiorespiratory interaction since Hales described changes in blood pressure related to the respiratory pattern in a horse (Hales 1733). Since then there has been much significant work on the cardio-respiratory interaction (e.g. Angelone and Coulter 1964, Davies and Nielson 1966, Hirsh and Bishop 1981). However, studies of the interactions between the other processes involved in the dynamics of the cardiovascular system (CVS) are still in their infancy. It has recently been shown (Stefanovska and Bračič 1999, Stefanovska et al 1999a, 1999b, Bračič et al 2000, Bračič Lotrič et al 2000, Stefanovska et al this issue) that, on the time scale of one average circulation period, the cardiovascular system behaves in many ways as a set of 5 coupled, autonomous, nonlinear oscillators of widely differing frequencies.

There are many different approaches to the problem of modelling cardiovascular dynamics including e.g. modelling of the blood flow through the system of pipes, or modelling of the coupling mechanisms as a system of differential equations with delay (Hyndman et al 1971, Kitney et al 1985, deBoer et al 1987, Baselli et al 1988, Saul et al 1991, Starkee and Westerhof 1993, TenVoorde et al 1995, Seidel and Herzel 1995, Cavalcanti and Belardinelli 1996). The majority of models proposed interpret the observed oscillations in the heart rate and blood pressure in terms of nonlinearities and time delays. They concentrate mainly on short-time blood pressure control mechanisms, including respiratory oscillations and oscillations with period around 10s. Although these models provide deep insight into particular aspects of the CVS dynamics, their range of application is restricted. In particular, there are many characteristics revealed by time series analysis of the blood flow that they do not reproduce.

The approach discussed here - modelling the CVS as a system of coupled oscillators - is qualitatively different. It concentrates on the oscillatory nature of the dynamics on the time-scale of one average circulation period. It aims to develop the simplest possible model that is able to reflect the oscillatory character of the CVS dynamics and is capable of reproducing the main statistical and dynamical characteristics of the measured time series, including the form of the spectra, the variability of the heart rate and respiration, as well as of the blood flow and blood pressure, and the main experimentally observed features of synchronization phenomena (Hildebrandt 1987, Raschke 1987, Schäfer et al 1998, 1999, Bračič Lotrič and Stefanovska 2000, Mrovka et al 2000, Stefanovska et al 
2000).

In this paper, we present the results of numerical simulations of the proposed model, focusing on the type of couplings and the effect of noise on the dynamics of the oscillators' interaction. In section 2 we summarise very briefly the experimental observations that have been made to date, and which a satisfactory model may be expected to encompass. Section 3 discusses how the model is constructed, and Section 4 reports the results obtained from it under different assumptions about the coupling constants and the presence or absence of random fluctuations (noise) representing external influences and parts of the CVS that are not included explicitly in the model. Section 5 summarises the results and draws conclusions.

\section{Background}

The proposed model is based on the experimental observation that, on a time scale of minutes, there are five almost periodic oscillatory subsystems contributing to the regulation of blood flow (Stefanovska and Krošelj 1997, Bračič and Stefanovska, 1998a, 1998b, Stefanovska and Bračič 1999, Stefanovska et al 1999a, Kvernmo et al 1998, 1999, Bračič et al 2000, Bračič Lotrič et al 2000). Complex and multiple control mechanisms regulate both the frequency and the volume of the blood expelled by the heart in each cycle, allowing the organism to respond to the metabolic demand of different parts of the body, to adjust the pressure and the capacity of the CVS, and react to external excitations sensed by the autonomous nervous system. The experimental observations that, taken together as a whole, point unequivocally to the dynamical nature of the rhythmic activity of the CVS, can be summarized as follows -

- Timescales. Five timescales are involved, and they differ substantially from each other (average frequencies of $\sim 1.0,0.2,0.1,0.03$ and $0.01 \mathrm{~Hz}$ ). The physiological origin of the different frequency components are obvious in the case of $1 \mathrm{~Hz}$ (heart beat), and $0.2 \mathrm{~Hz}$ (respiration), reasonably well established in the case of $0.1 \mathrm{~Hz}$ (intrinsic myogenic activity of smooth muscles), but not yet known with certainty in the cases of $0.03 \mathrm{~Hz}$ (connected to the autonomous nervous system's control over the cardiovascular system) or $0.01 \mathrm{~Hz}$ (apparently associated with endothelial activity). However, they all seems to be characterized by autonomous oscillatory dynamics.

- Signal and site invariance. All 5 basic frequencies are observed in all measured signals characterizing cardiovascular dynamics, including: heart-rate variability (where, however, the heart-rate is by definition missing), blood flow, blood pressure, respiration and ECG.

- Time variations. Continuous wavelet analysis of the time series reveals that the characteristic frequencies and amplitudes of the oscillatory processes are themselves of a slowly-varying periodic nature. 
- Spectral width. In the amplitude-frequency plane each of the characteristic peaks averaged over time is relatively broad, and they seems to be superimposed on a broad (noisy?) spectral background. This suggests that stochastic (or possibly chaotic) components also play a role in the regulation of the cardiovascular dynamics.

- Combination frequencies. In cases where the characteristic or basic frequencies were almost constant (e.g. in the case of a patient in coma), their linear combinations were clearly demonstrated.

- Synchronization. Recent analysis of cardiorespiratory synchrograms (Schäfer et al 1998, 1999, Bračič Lotrič and Stefanovska 2000, Mrovka et al 2000, Stefanovska et al 2000) show that cardiac and respiratory oscillators can become synchronized in different $n: m$ synchronization regimes. Furthermore, it is now established that synchronisation phenomena can also arise between some of the other oscillators (Stefanovska and Hožič 2000).

- Zero Lyapunov exponent. In the blood flow of healthy subjects, one of the Lypunov exponents is always found to be equal to zero within experimental and numerical error. A zero value of one of the Lyapunov exponents suggests (Abarbanel et al, 1993) that the source is governed by differential equations, or by a finite time map. Based on this inference, we may take the zero exponent in the blood flow of healthy resting humans as implying the dominantly deterministic nature of the CVS signals.

We consider separately evidence derived, first, from the analysis of oscillations in heart rate variability (HRV), respiration, blood flow and blood pressure and, secondly, from the phenomenon of synchronization. The reason is that, as we shall see later, they provide complementary information required to determine the strength and type of coupling. Furthermore, the analysis of HRV is of well known clinical significance (Task Force of the ESC and the NASPE 1996), while initial investigations of synchronization between the heart rate and respiration are already demonstrating its potential importance in clinical measurements.

We emphasize that, if the idea of characterizing CVS dynamics in terms of a system of coupled oscillators is correct, then one of the general approaches to the analysis of such a system can indeed be based on an analysis of its synchronization (Rosenblum et al 2000, Pikovsky et al 2001). We mention here only a few experimental results -

- Cardiorespiratory synchronization in healthy subjects. Only short episodes of synchronization with a high locking ratio and high variability of the heart and respiration frequencies were observed in healthy subjects at rest. Their heart beat is around $1 \mathrm{~Hz}$ and the extent of its variability is age-dependent (Kaplan et al 1991, Jansen-Urstad et al 1997, Stein et al 1997, Yeragani et al 1997, Bračič Lotrič and Stefanovska 2000).

- A subject in coma. In a sedated subject in a critical state of coma, a very high heart frequency $(1.6 \mathrm{~Hz})$, with no variability and no synchronization, were observed 
(Stefanovska and Bračič 1999). Deprived of feedback control from the peripheral systems, the cardiac frequency resumes its autonomous value, similar to its state when the heart is taken out of the body.

- Cardiorespiratory synchronization in athletes. Athletes are characterized by a relatively low average heart frequency, high heart rate variability, and longer episodes of synchronization with lower locking ratios (Kvernmo et al 1998, Schäfer et al 1998, 1999).

- Type II diabetic subjects. Patients with type II diabetes mellitus demonstrate a high average heart rate, low heart rate variability (Bračič Lotrič et al 2000) and very long episodes of synchronization (Bračič Lotrič et al unpublished results).

- Ancsthesia. It has recently been shown that during anæsthesia in rats the cardiorespiratory system passes reversibly through a sequence of different phasesynchronized states as the anæsthesia level changes (Stefanovska et al 2000). The synchronisation state seems to provide a direct and objective measure of the depth of anæsthesia.

- Heart rate synchronized by a weak external forcing. Cardiorespiratory synchronization during paced respiration (Seidel and Herzel 1998) and synchronization of the heart with external periodic sounds or light signals (Anishchenko et al 2000) were also demonstrated.

All these observations demonstrate clearly that different states of the organism may correspond to different regimes of synchronization which, as already mentioned above, can be of clinical significance and strongly support the idea of modelling the CVS dynamics as a system of coupled oscillators.

This list of experimental evidence is far from complete. We mention just one more important observation. It was found that there is a statistically significant trend in the dependence of the time-averaged synchronization index (defined in Tass et al 1998, Rosenblum et al 2000) and the standard deviation of the HRV distribution (Bračič Lotrič and Stefanovska 2000). Control of the cardiovascular system thus seems to be maintained by a fine balance between variations of the eigenfrequencies of the oscillators, resulting in mutual amplitude and frequency modulation and, sometimes, mutual synchronization between the phases of the oscillators.

Moreover, an inverse relationship between the average cardiac frequency and the extent of its variation seems to exist. In resting subjects, the higher the cardiac frequency is, the lower is its variability (Tsuji et al 1996). Athletes lie at one extreme, with a low cardiac frequency (below $1 \mathrm{~Hz}$ ) and high heart rate variability, whereas a heart taken out of the body, or disconnected from the rest of the body by anæsthesia or sedatives, lies at the other extreme and is characterised by a high frequency (around $1.6 \mathrm{~Hz}$ ) and no variability. Synchronization seems to be involved in this interplay between the basic, average frequency and the extent of its variation: the more strongly the cardiac

ฯ The dynamics of the CVS in rats has been shown to posses similar features to those in humans, despite the cardiac and respiratory rhythms in rats being approximately 4 times faster than in humans. 
Modelling couplings among the oscillators of the cardiovascular system oscillations are synchronized to the respiratory oscillations, the less variable and the higher the cardiac frequency seems to be.

\section{The model}

The model synthesis was based upon the experimental observation (see above) that there are five main frequency components in all the physiological time series: blood pressure, blood flow, ECG and respiration. Accordingly, a system of five coupled oscillators to model the CVS dynamics was chosen. The set of equations used for the modelling was suggested in earlier publications of one of the authors (Stefanovska et al 1999, Stefanovska et al this issue). The physiological interpretation of oscillators, discussed above, is as follows -

\begin{tabular}{cl}
\hline Frequency $(\mathrm{Hz})$ & Physiological function \\
\hline 1 & Heart \\
0.2 & Respiration \\
0.1 & Myogenic activity \\
0.03 & Neurogenic activity \\
0.01 & Endothelial related metabolic activity \\
\hline
\end{tabular}

The basic unit in the model, corresponding to the autonomous part of an oscillator, is the Poincaré oscillator

$$
\begin{aligned}
& \dot{x}_{i}=-x_{i} q_{i}-\omega_{i} y_{i}+g_{x_{i}}(\mathbf{x}), \\
& \dot{y}_{i}=-y_{i} q_{i}+\omega_{i} x_{i}+g_{y_{i}}(\mathbf{y}), \quad q_{i}=\alpha_{i}\left(\sqrt{x_{i}^{2}+y_{i}^{2}}-a_{i}\right),
\end{aligned}
$$

where $\mathbf{x}, \mathbf{y}$ are vectors of oscillator state variables, $\alpha_{i}, a_{i}$, and $\omega_{i}$ are constants and $g_{y i}(\mathbf{y})$ and $g_{x i}(\mathbf{x})$ are linear coupling vectors. Although to some extent this choice is arbitrary, it is an oscillator that possesses the properties of structural stability, robustness and symmetry that are consistent with physiological understanding, and with the analyses of measured time series.

The activity of each subsystem is described by two state variables, the blood flow $x_{i}$, and the velocity of flow $y_{i}$, where $i=1$ is generated by the heart, $i=2$ by respiration, $i=3$ by the myogenic oscillator, $i=4$ by the neurogenic oscillator, and $i=5$ by the metabolic oscillator. The mutual impact of the subsystems is taken into account as coupling terms. Additional effects resulting from the spatial distribution of some of the systems, such as the myogenic, neurogenic and endothelial related metabolic activity, and the effect of additional subsystems, acting on longer time scales than those considered, are taken into account as random noise.

The most nontrivial part of the model synthesis is the treatment of the couplings between oscillators. The type of couplings is currently unknown for most of the relationships between the subsystems. In the model, therefore, linear couplings are introduced. This type of coupling can be further justified by the observation of the same basic frequency components in all the physiological signals characterizing the CVS 
dynamics, and by the fact that only very small amplitude combinatorial components are observed in these signals.

It is well-known that time delays are very important in the description of physiological systems and are often used in modelling various parts of the cardiovascular system (Hyndman et al 1971, Kitney et al 1985, deBoer et al 1987, Baselli et al 1988, Saul et al 1991, Starkee and Westerhof 1993, TenVoorde et al 1995, Seidel and Herzel 1995, Cavalcanti and Belardinelli 1996). In our approach we understand the blood distribution system as one in which continuous information about every subsystem, acting with essentially different frequencies, is provided and fed back via coupling terms. There are thus delays corresponding to the time scale on which the particular subsystem acts. A number of delays therefore exist in the system and at this stage, again for the sake of simplicity of the proposed model, we effectively introduce them via additive coupling terms, corresponding to the time scales of the coupled subsystems. In this way not only few, but multiple delays are introduced into the system.

In some cases the sign of the coupling term can be determined from existing physiological knowledge and experimental observations. Direct modulation of the heart frequency by the respiration, and clear evidence of the corresponding combinational frequencies in the Fourier spectra for the subject in coma, suggest that unidirectional parametric coupling, i.e. cardiac frequency modified by respiration, but not vice versa, has to be included in the model. Moreover, the experimental evidences also suggest that most of the couplings are not symmetrical. Additional difficulties in the model synthesis are related to the fact that the myogenic, neurogenic and metabolic activities cannot be measured directly in physiological experiments using noninvasive techniques.

In what follows we will first introduce the model with linear coupling terms and discuss briefly their physiological relevance. We then consider the case of unidirectional parametric coupling and allow the cardiac frequency to be modulated by the rest of the system.

The function of the heart can be presented as

$$
\begin{aligned}
& \dot{x}_{1}=-x_{1} q_{1}-y_{1} \omega_{1}+\eta_{2} x_{2}-\eta_{3} x_{3}-\eta_{4} x_{4}+\eta_{5} x_{5}-\eta_{6}\left(\Phi_{1}-\Phi_{2}\right) \\
& \dot{y}_{1}=-y_{1} q_{1}+x_{1} \omega_{1}+\eta_{2} y_{2}-\eta_{3} y_{3}-\eta_{4} y_{4}+\eta_{5} y_{5}
\end{aligned}
$$

where $\eta_{i}$ are coupling terms. Respiratory sinus arrhythmia (see Section 1) refers to frequency modulation of the heart rate: the heart beats faster during inspiration and slower during expiration. Comparable modulation phenomena can also be observed in each part of the circulatory system: the amplitude of the flow component resulting from the heart beat in the peripheral blood flow is modulated by the frequency of respiration. It was shown that the coupling between the heart and respiration enables both modulation and synchronisation to occur. At the moment just a single term $\left(\eta_{2}\right)$ is used in the model for the cardio-respiratory coupling, although it is evident that in reality several mechanisms are involved.

Negative influences of the myogenic $\left(-\eta_{3}\right)$ and neurogenic system on the heart $\left(-\eta_{4}\right)$ are considered. The autonomous nervous system processes the information sensed by 
receptors and continuously adjusts the heart rate. The heart is innervated by both divisions of the autonomous nervous system - the sympathetic increases, and the parasympathetic decreases, the frequency and amplitude of myocardial contractions. Parasympathetic tone predominates in healthy resting humans. Increased metabolic activity results in an increased heart rate: a positive control loop is therefore assigned to the metabolic system $\left(\eta_{5}\right)$.

The difference between the inflow $\left(\Phi_{1}\right)$ on the arterial side and the outflow $\left(\Phi_{2}\right)$ to the venous side also contributes to the flow of blood generated by the heart. The dynamics of each of the other oscillators and their couplings at any point of the cardiovascular system can be described in a similar way (see Stefanovska et al this issue).

However, the cardiac contribution to the flow at each point results not only from the influences of local regulatory mechanisms, but also from the integration of the pressure $(P)$ and flow $(\Phi)$ values along the entire system. A global effect of the pressure on the myogenic and the neurogenic activity, and of the flow on metabolic activity, is also to be expected. Therefore, in (Stefanovska et al 1999b, Stefanovska et al this issue) the model was extended to describe the activity of the oscillators that regulate the flow of blood along the cardiovascular system

$$
\begin{aligned}
& \dot{x}_{1}=-x_{1} q_{1}-y_{1} \omega_{1}+\eta_{2} x_{2}-\eta_{3} x_{3}-\eta_{4} x_{4}+\eta_{5} x_{5}+ \\
&+\eta_{6} \int_{0}^{l} P(z, t) d z+\eta_{7} \int_{0}^{l} \Phi(z, t) d z \\
& \dot{y}_{1}=-y_{1} q_{1}+x_{1} \omega_{1}+\eta_{2} y_{2}-\eta_{3} y_{3}-\eta_{4} y_{4}+\eta_{5} y_{5}, \\
& \dot{x}_{2}=-x_{2} q_{2}-y_{2} \omega_{2}+\theta_{4} x_{4}+\theta_{5} x_{5}+ \\
&+\theta_{6} \int_{0}^{l} P(z, t) d z+\theta_{7} \int_{0}^{l} \Phi(z, t) d z \\
& \dot{y}_{2}=-y_{2} q_{2}+x_{2} \omega_{2}+\theta_{4} y_{4}+\theta_{5} y_{5}, \\
& \dot{x}_{3}=-x_{3} q_{3}-y_{3} \omega_{3}+\gamma_{4} x_{4}-\gamma_{5} x_{5}-\gamma_{6} P \\
& \dot{y}_{3}=-y_{3} q_{3}+x_{3} \omega_{3}+\gamma_{4} y_{4}-\gamma_{5} y_{5}, \\
& \dot{x}_{4}=-x_{4} q_{4}-y_{4} \omega_{4}-\rho_{2} x_{2}+\rho_{3} x_{3}-\rho_{5} x_{5}-\rho_{6} P \\
& \dot{y}_{4}=-y_{4} q_{4}+x_{4} \omega_{4}-\rho_{2} y_{2}+\rho_{3} y_{3}-\rho_{5} y_{5}, \\
& \text { and } \\
& \dot{x}_{5}=-x_{5} q_{5}-y_{5} \omega_{5}+\sigma_{2} x_{2}-\sigma_{3} x_{3}-\sigma_{4} x_{4}+\sigma_{6} \Phi \\
& \dot{y}_{5}=-y_{5} q_{5}+x_{5} \omega_{5}+\sigma_{2} y_{2}-\sigma_{3} y_{3}-\sigma_{4} y_{4} .
\end{aligned}
$$

where $\eta_{i}, \theta_{i}, \gamma_{i}, \rho_{i}$ and $\sigma_{i}$ are coupling terms and $\Phi$ and $P$ are the flow and pressure at any point of the circulatory system. The flow $\Phi$ is generated by the heart, so that $\Phi(0, t)=x_{1}(t)$. The pressure $P$ is generated by the lungs and $P(0, t)=x_{2}(t)$. We have assumed the flow to be in the $z$ direction. The blood returns to the right atrium of the 
heart at pressure of $0 \mathrm{~Pa}$ and at almost $0 \mathrm{~Pa}$ from the pulmonary vein to the left atrium. There, the value of $P(l, t)$ becomes 0 . By analogy, we may assume the value for $\Phi$ at this boundary condition $\Phi(l, t)=0$. The blood flow along the circulatory system can be described as

$$
\begin{aligned}
& \frac{\partial \Phi}{\partial t}=-\kappa_{1} \frac{\partial P}{\partial z}-\kappa_{2} x_{5} \quad ; \kappa_{i}>0 \\
& \frac{\partial P}{\partial t}=-\mu_{1} \frac{\partial \Phi}{\partial z}+\mu_{2} x_{3}+\mu_{3} x_{4} ; \mu_{i}>0
\end{aligned}
$$

where $\kappa_{i}, \mu_{i}$ are control parameters.

\section{Preliminary results of numerical simulations}

In the preliminary stage of modelling, presented in this paper, the main aim was to obtain some initial insight into the numerical characteristics of the model and, in particular -

- to examine the basic principles used in the model synthesis;

- to verify the ability of this model to reproduce the main features observed in the time series analysis and in the analysis of synchronization;

- to check how sensitive the model is to changes in the oscillator parameters and in the coupling vectors, and

- to determine the extent to which the strength and direction of coupling can be identified by comparison of the measured and model time series.

We have carried out a digital simulation of the model, using a high-speed pseudo-random generator (Marsaglia and Tsang 1984) for the increment $\Delta x_{1}$. Time series for each of the oscillators were generated by a sampling frequency of $100 \mathrm{~Hz}$. For each set of parameters 82 min records were generated. To simplify manipulations with the multi-dimensional coupling vector we have built a MatLab based graphical user interface providing for real time control of all the parameters of the system.

The flow component of each of the oscillators can be presented graphically after each run for a selected set of parameters. Here, we focus on the cardiac and respiratory components. Since the time series of the cardiac flow is, to a great extent, comparable to heart rate variability, we also focus on its spectral content. At this stage, however, as we shall see below, the simplified model is only capable of reproducing time variability of the heart rate to a limited extent. A windowed Fourier transform is used for calculation of the spectra. A time window of $246 \mathrm{~s}$ was used for estimation of the power spectrum, and the calculation was repeated for 20 windows, with no overlaps between them. The power spectrum presented here is thus a time average over the spectra obtained within 20 windows. In reality, all measured signals contain time variable frequency content and for estimation of their spectral components we need time-frequency methods, i.e. the wavelet transform. 
Time variability was taken into account in synchronization analysis between the cardiac and respiratory oscillators. The synchronization or mutual adjustment of the cardiac and respiratory rhythms was analysed using the recently proposed method for the detection of synchronization between irregular and non-stationary oscillators (Pikovsky et al 2001). The relative phase of the heartbeats within one respiratory cycles $\Psi_{1}$, was calculated for each set of parameters using the marker events method. The occurrence of synchronization was examined in a synchrogram, constructed by plotting the normalised relative phase of the cardiac component within 1 respiratory cycle, $\Psi_{1}=\frac{1}{2 \pi}\left(\Phi_{r}\left(t_{k}\right) \bmod 2 \pi\right)$, where $t_{k}$ is the time of $k$ th heartbeat and $\Phi_{r}$ is the instantaneous phase of respiration. Horizontal plateaus in the synchrogram are then interpreted as representing a constant phase difference, and thus the occurrence of phase synchronization.

\subsection{Linear couplings}

The set of equations (3)-(7) described in the previous section includes coupling with the multiple degrees of freedom of the CVS accumulated in the blood pressure and blood flow. However, here we start with a simplified version of the model, taking into account the description of the flow at a point in the cardiovascular system, of the form (2) introduced above for the cardiac oscillator. Hence, in the first stage, no effect of multiple degrees of freedom was taken into account. Our preliminary analysis of the model shows that it is possible to identify at least one set of parameters that enables the characteristic features of the time series and power spectra to be reproduced. We emphasize that whether or not this parameter set is unique remains for the present an open question. However, our initial results have already suggested some further experiments to try to clarify the matter. For the present studies, the model parameters were set as follows -

$\alpha_{1}=1.0, a_{1}=0.5, f_{1}=1.1 \mathrm{~Hz}, \eta_{2}=-\eta_{3}=-\eta_{4}=\eta_{5}=0.5$,

$\alpha_{2}=1.0, a_{2}=1.0, f_{2}=0.36 \mathrm{~Hz}, \theta_{4}=\theta_{5}=0.1$,

$\alpha_{3}=1.0, a_{3}=1.0, f_{3}=0.1 \mathrm{~Hz}, \gamma_{4}=\gamma_{5}=0.1$,

$\alpha_{4}=1.0, a_{4}=1.0, f_{4}=0.04 \mathrm{~Hz}, \rho_{2}=\rho_{3}=\rho_{5}=0.1$, and

$\alpha_{5}=1.0, a_{5}=0.5, f_{5}=0.01 \mathrm{~Hz}, \sigma_{2}=\sigma_{3}=\sigma_{4}=0.1$.

In Figure 1 results corresponding to the presence of linear coupling terms only are shown. It can be seen that the power spectrum of the oscillations in the blood flow, generated by the heart, reproduce the main characteristic features observed experimentally in the peripheral blood flow signal measured by a laser Doppler technique, including the position and relative intensities of the peaks. However, the widths of the peaks, and correspondingly the variability of the heart and respiration rates, are much smaller then those observed experimentally. In particular, no epochs of synchronization are observed in the cardio-respiratory synchrograms.

Here we note that the variation of the coupling parameters and/or natural 

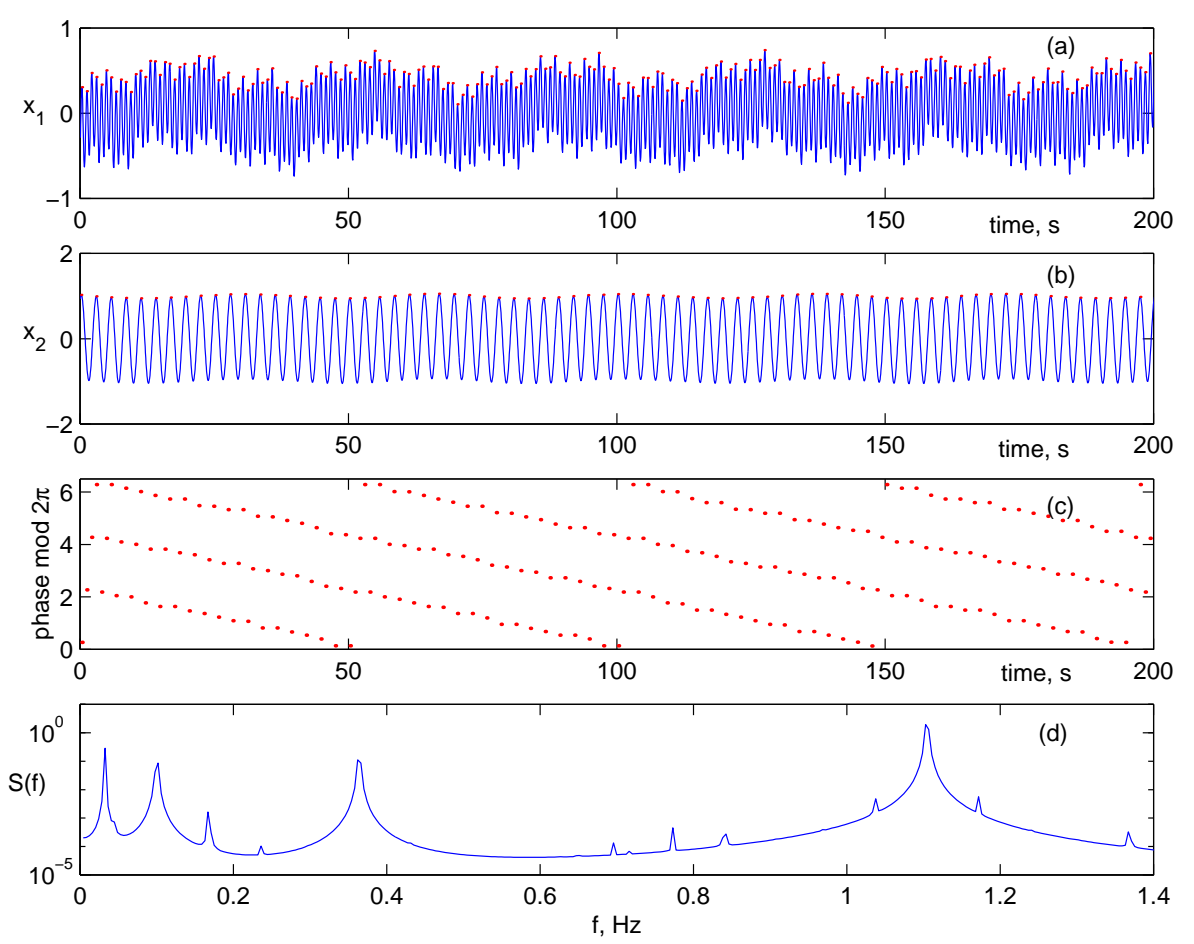

Figure 1. The results of modelling with deterministic linear couplings, in the absence of fluctuations. (a) and (b) The time series showing the rhythmic activities of the cardiac and respiratory oscillators, $x_{1}$ and $x_{2}$. (c) The corresponding cardiorespiratory synchrogram. (d) Power spectrum of the time series for the flow generated by cardiac oscillator, $x_{1}$. The component $x_{1}$ is comparable to the heart rate variability signal, which is obtained from the instantaneous cardiac frequency, determined by two successive R-peaks in the ECG signal.

frequencies will also give rise to synchronization in the system. However, motivated by the experimental observation of short episodes of synchronization (Shäffer et al 1998, 1999, Bračič Lotrič and Stefanovska 2000), we will concentrate on the non-synchronized state. We are interested in establishing whether or not it is possible to reproduce in numerical simulations the regime where short epochs of synchronization occur.

\subsection{Linear couplings in the presence of fluctuations}

In the next step, couplings with multiple degrees of freedom accumulated in the blood pressure and blood flow were added in an attempt to introduce the globally distributed effects of the CVS. However, at this stage equations (3)-(7) were not modelled numerically to their full extent and $\eta_{6}=\eta_{7}=\theta_{6}=\theta_{7}=\gamma_{6}=\rho_{6}=\sigma_{6}=0$. Rather, a fluctuational term was added to the cardiac oscillations $(2)$

$$
\begin{aligned}
& \dot{x}_{1}=-x_{1} q_{1}-2 \pi f_{1} y_{1}+g_{x_{1}}(\mathbf{x})+\xi(t), \\
& <\xi(t)>=0, \quad<\xi(t) \xi(0)>=D \delta(t),
\end{aligned}
$$

Thus, all influences of the rest of the system are lumped in the fluctuational term $\xi(t)$. The addition of the random term, very interestingly, resulted in epochs of 

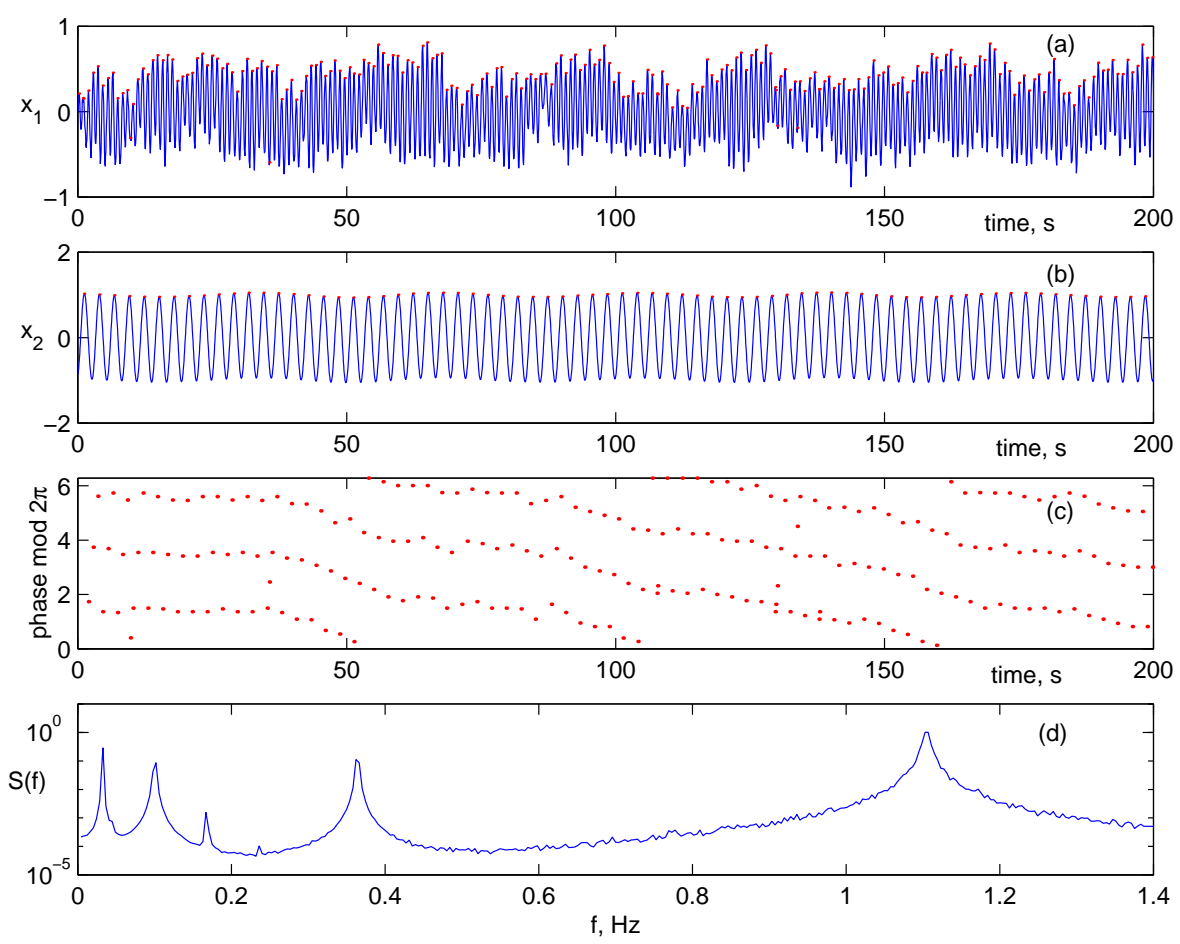

Figure 2. Results of modelling with linear couplings, in the presence of fluctuations. (a) and (b) The time series showing the rhythmic activities of the cardiac and respiratory flow components. (c) The corresponding cardio-respiratory synchrogram. (d) Power spectrum of oscillation in the blood flow generated by the cardiac activity.

synchronization in the cardio-respiratory synchrograms. Without noise, only the standard regimes of phase-locking, phase modulation, and their interplay, could be observed. However, the heart rate variability remains very small suggesting that probably parametric coupling also plays an important role in the cardiac activity and the activity of other cardiovascular oscillators.

\subsection{Parametric couplings}

Just for comparison we show in Figure 3 results corresponding to purely parametric deterministic coupling of the heart rhythmic activity to the rest of the system in the form

$$
\begin{aligned}
& \dot{x}_{1}=-x_{1} q_{1}-y_{1}\left(\omega_{1}+\eta_{2} x_{2}-\eta_{3} x_{3}-\eta_{4} x_{4}+\eta_{5} x_{5}\right) \\
& \dot{y}_{1}=-y_{1} q_{1}+x_{1}\left(\omega_{1}+\eta_{2} y_{2}-\eta_{3} y_{3}-\eta_{4} y_{4}+\eta_{5} y_{5}\right),
\end{aligned}
$$

It can clearly be seen that although the heart rate variability can be increased dramatically the power spectra and the cardio-respiratory synchrogram still differ substantially from those observed experimentally.

Synchronisation of a noisy van der Pol oscillator with modulated natural frequency has already been analysed in the context of the cardiorespiratory interaction (Shäffer at al 1999). Frequency locking was obtained only if the amplitude of modulation exceeded 

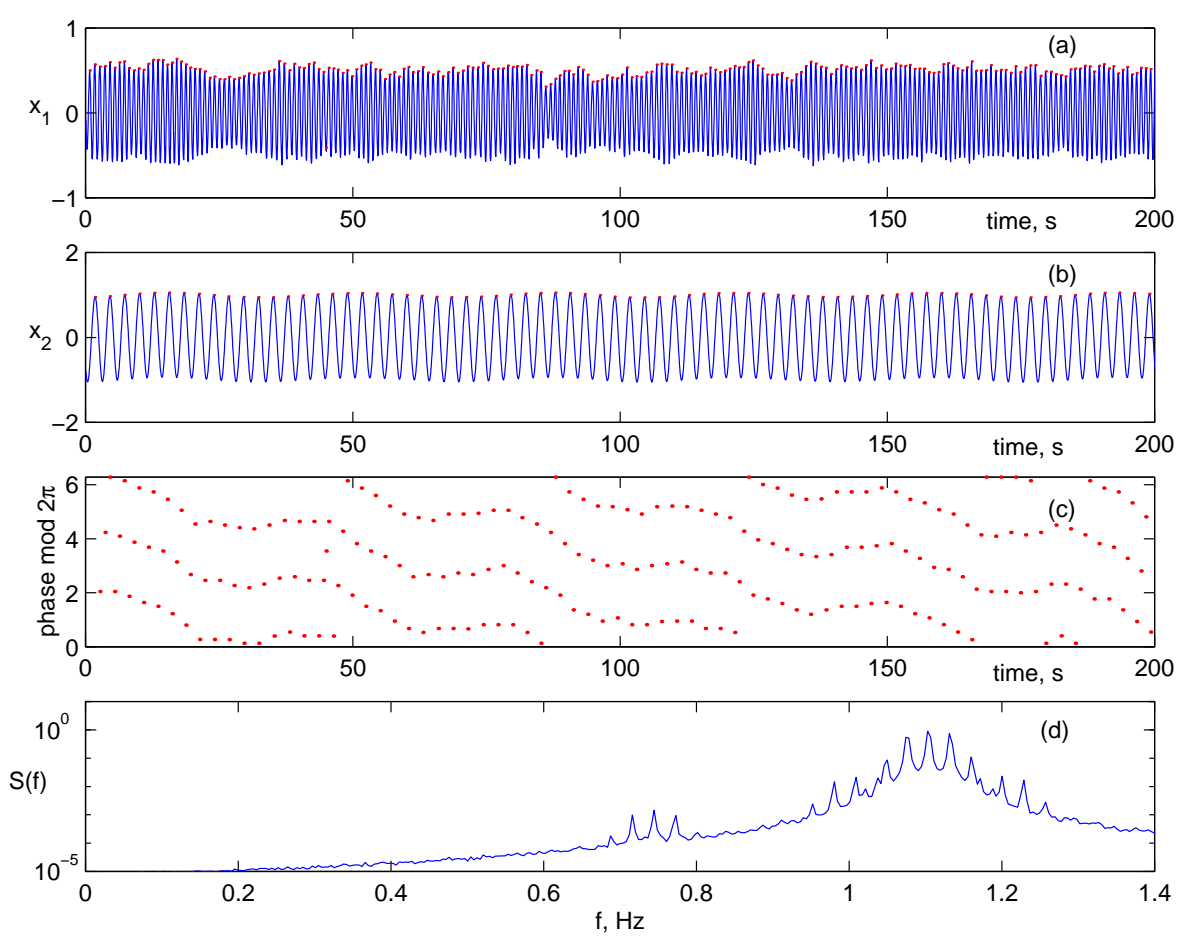

Figure 3. The results of the modelling with pure deterministic parametric couplings. (a), (b), (c), and (d) are the same as in figures 1 and 2.

some threshold value. Moreover, the presence of noise smeared this locking effect, while quasiperiodic modulation, as also considered in our case, resulted in frequency locking without phase locking.

Modulation and synchronisation may or may not coexist in parametrically coupled systems in presence of noise. This was also observed for the cardiorespiratory interaction, where generally the intensity of synchronisation appears to be inversely proportional to the modulation of cardiac frequency by the respiratory related rhythm (Bračič Lotrič and Stefanovska, 2000). However this relation is changed in subjects with cardiovascular disease suggesting that this two mechanisms are essential for the functioning of the cardiovascular system. However, whether the parametric coupling enables this interplay to occur remains to be clarified.

\section{Summary and conclusions}

The preliminary analysis of the model, presented above, has been restricted to the cardio-respiratory interaction on account of two main reasons. On one hand, there is a number of experimental results related to this type of interaction including e.g. investigation of the respiratory-sinus arrhythmia and cardio-respiratory synchronization. On the other hand, it is only these two high frequency signals of the model that can be related directly to the experimentally observable variables. However, even at this early stage of the investigations, some important conclusions can be reached about the type 
of coupling and the role of fluctuations.

The results obtained suggest that the type and strength of the linear coupling in our model can be determined quite accurately through comparison of the observed and simulated time series. Comparison of the experimental data with time series obtained from the model suggests, in particular, that in order to reproduce the main dynamical features of the cardiac rhythmic activity both linear and parametric couplings have to be taken into account, with linear coupling playing the dominant role. This comparison also shows that the fluctuations play an important role in the CVS dynamics. In particular, fluctuations are responsible (at least in part) for the experimentally observed and numerically reproduced broadening of the main spectral peaks and for a number of regimes of synchronization.

Here we note that the model is capable of reproducing the different patterns of synchrogrammes observed experimentally including e.g. the standard phase locking regime and the regime of continuously varying phase both in the presence and in the absence of noise. We were specifically interested to establish whether it is possible to reproduce in numerical simulations the regime of short epochs of synchronisation observed in the experiment (see Fig. 4 in Stefanovska et al this issue). It turns out that such regime can be reproduced numerically only in the presence of fluctuations. To do so one can tune e.g. cardiac and respiration frequencies close to, but slightly outside, the regime of phase locking. By adding fluctuations to the cardiac oscillator it is then possible to induce short epochs of synchronization as shown in the Fig. 2.

A qualitative explanation of this phenomenon within the framework of the current model may be as follows. In a first approximation, the interaction between the heart and respiratory oscillations is due to a unidirectional influence of the respiratory oscillator on the heart rhythm. The effect of the coupling to the rest of the system can be taken into account by allowing for the fluctuations. This simplified situation can be modelled as a singled out nonlinear oscillator driven by periodic and random forces. The model can be further simplified. By introducing a slowly varying phase difference between the phase of oscillator and the phase of the driving force $\phi(t)=\Phi(t)-\Psi(t)$, and by assuming a constant amplitude approximation (Stratanovich 1963), we have

$$
\dot{\phi}=\Delta+K \sin (\phi)+\psi(t)
$$

where $\Delta$ is the detuning $n \omega_{1}-m \omega_{2}, K$ is a function of the system parameters, $\phi$ is the slowly varying phase of the cardiac oscillations and $\psi$ is a new random zero-mean Gaussian variable.

It can be seen from (11) that small values of $\Delta$ correspond to a small slope of the wash-board potential corresponding to (11) and to the locking of the phase $\phi$ at the bottom of one of the potential wells. On the other hand relatively large values of $\Delta$ correspond to a large slope of the wash-board potential, to the disappearance of the local potential well and to the absence of synchronization. In the presence of weak noise two effects can be expected. For a small value of $\Delta$, noise can induce transitions of the phase between different potential wells. In this regime the system will stay mainly in a 
state of synchronization but, from time-to-time, phase steps $\propto 2 \pi$ will occur. Another possible regime for relatively large (but not too large) values of $\Delta$ is that the system may stay mainly in the desynchronized state modulated by noise. If however large rare fluctuations effectively decrease the value of $\Delta$, short epochs of synchronization will be observed. This hypothesis can in principle be verified using methods for the direct analysis of the fluctuational dynamics (Luchinsky et al 1998), as will be discussed in a future publication.

We conclude that our preliminary results support the model of five coupled oscillators suggested and justified in earlier physiological experiments (Stefanovska and Krošelj 1997, Bračič and Stefanovska, 1998a, 1998b, Stefanovska and Bračič 1999a, Stefanovska et al 1999a, 1999b, Kvernmo et al 1998, 1999, Bračič et al 2000, Bračič Lotrič et al 2000). In particular, the model is able to reproduce some basic features of the cardio-respiratory interactions observed in physiological time series including e.g. heart rate variability, synchronization phenomena and the general shape of the power spectra.

Moreover, the preliminary analyses suggest that the information obtained from these time series is complementary to that from the physiological measurements, in that it will allow one to determine at least approximately the type and strength of coupling.

One of the important conclusions is that the interplay between the synchronization and frequency modulation can be taken into account by adding noise to the system. The latter corresponds to the fact that, in the simplified version of the model, the cardiac and respiratory rhythmic activities are coupled only to the local low frequency oscillations taken at some arbitrary point of the cardiovascular system. However, in reality there is an ensemble of infinitely many such local low frequency oscillators characterized by the distribution over oscillator's frequencies, amplitudes and phases. This could be taken into account e.g. by introducing ensemble-averaged values of the low frequency components $\bar{x}_{3}, \bar{x}_{4}, \bar{x}_{5}, \bar{y}_{3}, \bar{y}_{4}, \bar{y}_{5}$ into the equations of motion for the cardiac and respiratory oscillations and by allowing for fluctuations of these values within the range determined by their standard deviations from the averaged values found from the times series analyses.

The cardiovascular system, as probably all living systems, is characterised by the presence of large numbers of degrees of freedom and, remarkably, the ability to effect a natural grouping of the degrees of freedom and time scales. It appears that the phase locking of coupled oscillators provides a means to bind degrees of freedom.

\section{Acknowledgements}

The research of A Stefanovska was supported by the Royal Society of London and the Slovenian Ministry of Science and Technology; and that of D G Luchinsky and P V E McClintock by the Engineering and Physical Sciences Research Council (UK). 
Modelling couplings among the oscillators of the cardiovascular system

\section{References}

Abarbanel H D I, Brown R, Sidorowich J J, Tsimring L Sh 1993 The analysis of observed chaotic data in physical systems Rev. Mod. Phys. 65, 1331

Angelone A and Coulter N A 1964 Respiratory sinus arrhyrhmia: a frequency dependent phenomenon J. Appl. Physiol. 19479

Anishchenko V S, Balanov A G, Janson N B, Igosheva N B, and Bordyugov G V 2000 Synchronisation of heart rate by sound and light pulses Stochastic and Chaotic Dynamics in the Lakes eds D S Broomhead et al (Melville, New York: AIP Conference Proceedings)

Berne R M and Levy M N eds 1998 Physiology (Mosby, St. Louis, Missouri: Mosby)

Bračič M and Stefanovska A 1998a Nonlinear dynamics of blood flow studied by Lyapunov exponents Bull. Math. Biol. 60417

Bračič M and Stefanovska A 1998b Wavelet based analysis of cardiovascular dynamics Bull. Math. Biol. 60919

Bračič M, McClintock P V E, and Stefanovska A 2000 Characteristic frequencies of the human blood distribution system Stochastic and Chaotic Dynamics in the Lakes eds D S Broomhead et al (Melville, New York: AIP Conference Proceedings)

Bračič Lotrič M and Stefanovska A 2000 Synchronisation and modulation in the human cardiorespiratory system Physica A 283451

Bračič Lotrič M, Stefanovska A, Štajer D, and Urbančič-Rovan V 2000 Spectral components of heart rate variability determined by wavelet analysis Physiol. Meas. 21441

Cavalcanti A and Belardinelli E 1996 Modelling cardiovascular variability using differential delay equation IEEE Trans. Biomed. Eng. 43982

deBoer R W, Karemaker J M, and Strackee J 1987 Hemodynamic fluctuations and baroreflex sensitivity in humans: A beat-to-beat model. Am. J. Physiol. 253 H680

Davis C T M and Neilson J M M 1967 Sinus arrhythmia in man at rest J. Appl. Physiol. 22947

Haken H 1983 Advanced Synergetics (Berlin:Springer)

Hales S 1733 Statical Essays II, Haemastaticks (London: Innings Manby)

Hildebrandt G 1987 The autonomous time structure and its reactive modifications in the human organism Temporal Disorder in Human Oscillatory Systems eds L Resing et al (Berlin:Springer)

Hirsch J A and Bishop B 1981 Respiratory sinus arrhythmia in humans: how breathing pattern modulates heart rate Am. J. Physiol. (Heart Circ. Physiol. 10) 241 H620

Hyndman B W, Kitney R I, and Sayers B McA 1971 Spontaneous rhythms in physiological control systems Nature 233339

Jensen-Urstad K, Storck N, Bouvier F, Erickson M, Lindblad L E, and Jensen-Urstad M 1997 Heart rate variability in healthy subjects is related to age and gender Acta Physiol. Scand. 160235

Kaplan D T, Furman M I, Pincus S M, Ryan S M, Lipsitz L A, and Goldberger A L 1991 Aging and the complexity of cardiovascular dynamics Biophys. J. 59945

Kitney R I, Fulton T, McDonald A H, and Linkens D A 1985 Transient interactions between blood pressure, respiration and heart rate in man J. Biomed. Eng. 7

Kuramoto Y 1984 Chemical Oscillations, Waves, and Turbolence (Berlin:Springer)

Kvernmo H D, Stefanovska A, Bračič M, Kirkebøen K A, and Kvernebo K 1998 Spectral analysis of the laser Doppler perfusion in human skin before and after exercise Microvasc. Res. 56173

Kvernmo H D, Stefanovska A, Kirkebøen K A, and Kvernebo K 1999 Oscillations in the human cutaneous blood perfusion signal modified by endothelium-dependent and endothelium-independent vasodilators Microvasc. Res., 57298

Luchinsky D G, McClintock P V E, and Dykman M I 1998 Analogue studies of nonlinear systems Reports on Progress in Physics, 61889

Marsaglia G and Tsang W W 1984 A fast, easily implemented method for sampling from decreasing or symmetric unimodal density-functions, SIAM J. Sci. Stat. Comput. 5349

Mrowka R, Patzak A, and Rosenblum M G 2000 Quantitative analysis of cardiorespiratory 
Modelling couplings among the oscillators of the cardiovascular system

synchronization in infants, Int. J. of Bifurcation and Chaos 10 (11) 2479

Pikovsky A, Rosenblum M, and Kurths J 2001 Synchronization - A Universal Concept in Nonlinear Sciences (Cambridge:Cambridge University Press)

Rosenblum M G, Pikovsky A S, Schäfer C, Tass P, and Kurths J, 2000 Phase Synchronization: From Theory to Data Analysis, Handbook of Biological Physics, eds Moss F and Gielen S, (Elsevier Science) 279

Raschke F 1987 Coordination in the circulatory and respiratory systems. Temporal Disorder in Human Oscillatory Systems eds L Resing et al (Berlin:Springer)

Saul J P, Berger R D, Albrecht P, Stein S P, Hui Chen M and Choen R J 1991 Transfer function analysis of the circulation: unique insights into cardiovascular system Am. J. Physiol. $261 \mathrm{H} 12321$

Schäfer C, Rosenblum M G, Kurths J, and Abel H H 1998 Heartbeat synchronised with respiration Nature 293239

Schäfer C, Rosenblum M G, Abel H H, Kurths J 1999 Synchronization in human cardiorespiratory system Physical Review E 60857

Seidel H and Herzel H 1995 Modelling heart rate variability due to respiration and baroreflex Dynamical phenomena in living systems eds E Mosekilde and O G Mouritsen (Berlin:Springer)

Seidel H and Herzel H 1998 Analysing entrainment of heartbeat and respiration with surrogates IEEE Eng. Med. Biol. Mag. 17

Starkee J and Westerhof N eds 1993 The physics of heart and circulation (Bristol:IOP) Ljubljana.

Stefanovska A and Krošelj P 1997 Reconstructing cardiovascular dynamics using correlation integral and frequency analysis Open Systems and Information Dynamics 4457

Stefanovska A and Bračič M 1999 Physics of the human cardiovascular system Contemporary Physics 4031

Stefanovska A, Bračič M, and Kvernmo H D 1999a Wavelet analysis of oscillations in the peripheral blood circulation measured by laser Doppler technique IEEE Trans. Biomed. Eng. 461230

Stefanovska A, Strle S, Bračič M, and Haken H 1999b Model synthesis of the coupled oscillators which regulate human blood flow dynamics Nonlinear Phenomena in Complex Systems 272

Stefanovska A and Hožič 2000 Spatial synchronization in the human cardiovascular system Progr. Theor. Phys. Suppl. 139270

Stefanovska A, Haken H, McClintock P V E, Hožič M, Bajrovič F, and Ribarič S 2000 Observations of reversible phase transitions in the cardio-respiratory system Phys. Rev. Lett. 854831

Stefanovska A, Bračič Lotrič M, Strle S, and Haken H 2001 The cardiovascular system as coupled oscillators? Physiol. Meas. this issue

Stein P K, Kleiger R E, and Rottman J N 1997 Differing effects of age on heart rate variability in men and women Am. J. Cardiol. 80302

Stratonovich R 1963 Topics in the Theory of Random Noise (New Yuork:Gordon and Breach)

Strogatz S H 1994 Nonlinear Dynamics and Chaos (Reading:Addison-Wesley)

Task Force of the ESC and the NASPE 1996 Standards of heart rate variability Eur. Heart J. 17354

Tass P, Rosenblum M G, Weule J, Kurths J, Pikovsky A S, Volkmann J, Schnitzler A, and Freund H-J 1998 Detection of n:m phase locking from noisy data: Application to magnetoencephalography, Phys. Rev. Lett. 813291

TenVoorde B J, Faes Th J C, Jansen T W J, Scheffer G J, and Rompelman O 1995 Respiratory modulation of blood pressure and heart rate studied with computer model of baroreflex control Computer Analysis of Cardiovascular Signals eds M Di Renzo et al (Amsterdam:IOS Press)

Tsuji H, Venditti F J, Manders E S, Evans J C, Larson M G, Feldman C L, Levi D 1996 Determinants of heart rate variability, JACC $\mathbf{2 8} 1539$

Yeragani V K, Sobolewski E, Kay J, Jampala V C, and Igel G 1997 Effect of age on long-term heart rate variability Cardiovasc. Res. $\mathbf{3 5} 35$ 